\title{
Utilización de la Escala de Empatía Médica de Jefferson en Residentes que realizan su especialización en un hospital peruano.
}

\author{
Use of the Jefferson's Medical Empathy Scale in Resident physicians in specialization training in a Peruvian \\ hospital.
}

\author{
Gualber V Mayo ${ }^{1}$, Eberth G Quijano ${ }^{1}$, Daniel A Ponce ${ }^{1}$, Ray Ticse ${ }^{1,2}$ \\ Identificación ORCID \\ Gualber V Mayo: https://orcid.org/0000-0001-8994-4371 \\ Eberth G Quijano: https://orcid.org/0000-0001-6218-8350 \\ Daniel A Ponce: https://orcid.org/0000-0002-6226-9289 \\ Ray Ticse: https://orcid.org/0000-0002-4568-5723
}

\section{RESUMEN}

La empatía es definida como la capacidad de comprender los sentimientos y emociones de los demás, basada en el reconocimiento del otro como persona similar o semejante. Aplicada a la relación médico-paciente, puede mejorar los resultados clínicos, por lo que es considerada una habilidad esencial en la capacitación de todo profesional médico. Objetivo: Identificar el nivel de empatía y factores relacionados en médicos residentes de áreas médicas y quirúrgicas realizando entrenamiento especializado en un hospital de Lima, Perú. Material y métodos: Se obtuvieron esenciales datos sociodemográficos y profesionales y se aplicó la Escala de Empatía Médica de Jefferson, conformada por 20 preguntas, con puntuación Likert de 1 a 7, puntaje mínimo de 20 y máximo de 140, y nivel de empatía directamente proporcional al puntaje obtenido. Resultados: Cien médicos residentes, $52 \%$ de sexo femenino constituyeron la muestra. La media del puntaje obtenido fue 112,88, con DE 14.51, mediana de 115 y coeficiente alfa de Cronbach de 0,82 . Los valores de los cuartiles fueron de 102, 115 y 124. Veintiséis por ciento de los médicos residentes de medicina, 19,2\% de cirugía, $10 \%$ de Gineco-obstetricia y 35\% de pediatría alcanzaron puntajes del cuartil superior. No se hallaron diferencias estadísticamente significativas al comparar el puntaje de los encuestados con cada una de las variables estudiadas. Conclusiones: Los médicos residentes de la especialidad de Pediatría presentaron puntajes más altos que el resto de especialidades, pero no se encontraron variables específicas significativas para explicar las diferencias detectadas en niveles de empatía.

PALABRAS CLAVE: Empatía, medicina, educación médica, Perú.

\section{SUMMARY}

Empathy is defined as the ability to understand the feelings and emotions of others, based on the recognition of the other as similar. Applied to the doctor-patient relationship can improve clinical outcomes, reason for which it is considered an essential ability in the training of every medical professional. Objective: To identify the level of empathy and related factors in resident physicians in medical and surgical specialized training in a hospital in Lima, Peru. Material and methods: Sociodemographic, personal and professional data were obtained and the Jefferson Medical Empathy Scale, consisting of 20 questions and a 1 to 7 Likert scoring type, was applied; with a minimum

1 Universidad Peruana Cayetano Heredia, Facultad de Medicina. Lima,Perú.

2 Unidad de Educación Médica, Facultad de Medicina, Universidad Peruana Cayetano Heredia. Lima, Perú. 
score of 20 and maximum of 140, the level of empathy is directly related to the obtained score obtained. Results: One hundred resident physicians, $52 \%$ of them female, were surveyed. The mean score obtained was 112.88 , with DE 14.51, median of 115, and a Cronbach's alpha coefficient of 0.82 . The values of the quartiles were 102, 115 and 124 . Twenty-six per cent of medical residents, $19.2 \%$ of surgery, $10 \%$ of gynecology-obstetrics and $35 \%$ of pediatrics reached higher values of the upper quartile. No statistically significant differences were found when comparing the respondents' scores with each of the identified variables. Conclusions: Residents of the Pediatrics specialty has higher scores than the rest of the specialties, although no specific significant variables were found to explain the differences between the empathy levels.

KEYWORDS: Empathy, medicine, medical education, Peru.

\section{INTRODUCCIÓN}

La empatía es definida como "la capacidad de comprender los sentimientos y emociones de los demás, basada en el reconocimiento del otro como similar" (1). De acuerdo con Morse et al., la empatía es un concepto multidimensional basado en cuatro pilares básicos que son emotivo, cognitivo, moral y conductual (2).

Aplicada al ámbito médico, se puede definir como un atributo cognitivo que incluye el entendimiento de experiencias, perspectivas del paciente como individuo, además de la capacidad de transmitir esta comprensión al paciente (3). Uno de los aspectos positivos de la empatía es que ayuda a afianzar la relación médico-paciente ya que genera mayor satisfacción por la atención recibida, se aporta mayor información al momento del interrogatorio, y se puede lograr acertar con el diagnóstico de forma más eficiente (4); además se ha podido observar que implica un mejor estado de bienestar mental para el profesional de la salud (5), menor estrés y burnout profesional (6). Sin embargo, la empatía puede estar afectada por el modelo de docencia vigente de algunos centros de formación que se basan solamente en la parte biológica del ser humano; o durante su aplicación, por la carga laboral y la falta de recursos del sistema de salud, limitando la enseñanza de temas relacionados a la empatía o su aplicación en el campo clínico. Esta también puede estar limitada por características propias de cada individuo como son el sexo, clase social, estado civil, tenencia o no de hijos, formación previa, experiencia laboral, situación familiar, emocional o sentimental $(6,7,8)$.

Se ha reportado que el sexo femenino o estar casado, está asociado a mayores niveles de empatía; mientras que otras características se asocian a menores niveles de empatía, como el no haber recibido formación en empatía $(3,9,10)$. Cabe resaltar que ninguna de estas asociaciones es concluyente, puesto que existen resultados discordantes aún en estudios con metodología similar, esta controversia se puede observar, por ejemplo, en variables como el tener o no hijos, ya que existen estudios que señalan que tener hijos afecta a la empatía de manera positiva, mientras que en otros, se observan mayores niveles de empatía en quienes no tienen hijos $(9,11)$.

Los profesionales de la salud deben tener formación integral, esto incluye tanto la experticia en conocimientos, así como competencias transversales como profesionalismo, trabajo en equipo y habilidades de comunicación efectiva, entre otras $(12,13)$. Sobre empatía, la mayoría de estudios fueron realizados en estudiantes de pregrado $(1,6,7)$; en países de habla no hispana existen algunos aplicados en médicos residentes $(9,14)$. En el Perú, sólo existen trabajos aplicados en estudiantes de pregrado de las carreas de medicina y estomatología $(15,16)$. La diferencia sustancial respecto a la población de médicos residentes yace en que estos últimos son responsables de la atención y prescripción de los pacientes; las decisiones y herramientas que utilicen tendrán mayor impacto en la salud de los pacientes (9).

La "Escala de Empatía Médica de Jefferson" ha sido validada en diferentes países alrededor del mundo, incluidos países de Latinoamérica y aplicada anteriormente en el Perú previa adaptación cultural, cuenta con validez de construcción, es decir que mide lo que pretende medir; además de ser convergente y discriminante, quiere decir, que las partes que lo conforman estén relacionadas entre sí; y que sus partes no guardan relación con temas distintos a la empatía. Además cuenta con coeficiente alfa de Cronbach que se sitúa entre 0,7 a 0,9 en sus diversas versiones y traducciones, lo que nos dice que es una escala de medida fiable y cada una de las preguntas que la conforman guardan correlación entre sí y con la totalidad del instrumento $(16,17)$. 
El objetivo de este estudio fue obtener una valoración del nivel de empatía en los médicos residentes que se encuentren realizando su especialización en un hospital docente en Lima, Perú; además de explorar las variables asociadas al nivel de empatía.

\section{MATERIAL Y MÉTODOS}

El Estudio es descriptivo transversal, fue aplicado a cien médicos residentes que realizan su programa de especialización en el Hospital Cayetano Heredia $(\mathrm{HCH})$ de Lima - Perú. El HCH forma parte del MINSA (Ministerio de Salud del Perú), es la sede docente principal de la Universidad Peruana Cayetano Heredia (UPCH).

Se reclutó a los participantes en cada uno de sus departamentos académicos correspondientes (medicina, cirugía, pediatría y gineco-obstetricia). Se incluyó como participante a todo médico residente que esté realizando su especialización durante febrero del 2018 en cada una de las áreas mencionadas y se excluyó a aquellos que no deseaban participar. La totalidad de los participantes respondió correctamente los cuestionarios.

Para medir el nivel de empatía se utilizó el cuestionario "Escala de Empatía Médica de Jefferson" en su versión "HP" (Health Professionals). El cual fue llenado de manera individual, confidencial y anónima por cada participante, en su servicio correspondiente. El cuestionario está compuesto de 20 preguntas, las cuales tienen un puntaje del 1 al 7 tipo Likert que corresponde desde "Totalmente en desacuerdo" a "Totalmente de acuerdo", respectivamente. Tiene un puntaje mínimo de 20 y máximo de 140, siendo el nivel de empatía directamente proporcional al puntaje obtenido. Este cuestionario define a la empatía en base a tres componentes: toma de perspectiva, atención con compasión y habilidad para "ponerse en los zapatos del paciente". Ha sido validada a nivel internacional como España y en países latinoamericanos que demuestran su grado de confiablidad adecuado (alfa de Cronbach de 0,7 a 0,9$)$.

Asimismo, se utilizó una ficha de recolección de datos junto al cuestionario para evaluar los posibles factores asociados al nivel de empatía como sexo, edad, estado civil, tenencia de hijos, año de residencia, años de egresado de pregrado, modalidad de ingreso a la residencia médica que puede ser libre, cautiva o destaque (estas últimas poseen relación laboral permanente previa con alguna institución pública de al menos cinco años) área de especialidad médica y si llevó algún taller o curso relacionado a la empatía.

Los datos fueron ingresados a una base de datos en el programa Microsoft Excel y se realizó el procesamiento y análisis de datos con el programa STATA v14.0. Se realizó el análisis descriptivo para determinar las medidas de frecuencia y de tendencia central de las variables. Se obtuvo los valores de los cuartiles 25, 50 y 75; con los cuales se tomó como "Bajo" los puntajes hasta el cuartil 25; "Medio", puntajes desde el cuartil 25 a 75 y "Alto" desde el cuartil 75 en adelante. Se realizó el test de Shapiro-Wilk para hallar normalidad o no en los resultados. De acuerdo a la distribución no normal de los datos se utilizaron pruebas no paramétricas. Utilizándose la prueba de U de Mann-Whitney para las variables dicotómicas, la prueba de Kruskal-Wallis para variables politómicas y la prueba Chi-cuadrado para los cuartiles. Asimismo, se usó la prueba de Correlación de Spearman para las variables continuas. Se consideró un $\mathrm{p}<0.05$ como significativo.

El estudio fue revisado y aprobado por el Comité Institucional de Ética en Investigación de la Universidad Peruana Cayetano Heredia.

\section{RESULTADOS}

Ingresaron al estudio cien médicos residentes. El $52 \%$ de los encuestados correspondieron al sexo femenino, una amplia mayoría (84\%) fueron solteros, así mismo se encontró que el $91 \%$ de los encuestados no tenían hijos (tabla 1). En cuanto al puntaje total obtenido, se observa que la media fue de 112,88 con DE (desviación estándar) de 14,51, la mediana resultó ser 115 y el coeficiente alfa de Cronbach fue 0,82 (tabla 2). Se aplicó la prueba de Shapiro-Wilk al nivel de empatía y se obtuvo que seguía una distribución no normal, por lo que se utilizaron pruebas no paramétricas (gráfico 1).

La modalidad de ingreso más común de los médicos residentes encuestados al programa de especialización fue la modalidad "plaza libre" $(89 \%)$, no encontrándose diferencia estadísticamente significativa $(\mathrm{p}=0,27)$ en cuanto al nivel de empatía entre quienes ingresaron por esta modalidad en comparación con aquellos que ingresaron por plaza cautiva o destaque (tabla 1).

Los encuestados fueron clasificados según el área de especialidad a la cual pertenece su programa de especialización, obteniéndose que el $50 \%$ pertenecen 
Tabla 1. Características de los médicos residentes que participaron en la evaluación de la empatía y sus factores relacionados según la Escala de Empatía Médica de Jefferson en un Hospital de Lima Perú.

\begin{tabular}{ccccc}
\hline & $\mathbf{n}$ & Mediana & Rangos Intercuartílicos & $\mathbf{p}$ \\
\hline Sexo & & & $(103,5-124,5)$ & 0,6094 \\
Femenino & 52 & 115 & $(103,5-123,5)$ & \\
$\quad$ Masculino & 48 & 115 & & \\
Estado civil & & & $(101,5-124)$ & 0,7207 \\
$\quad$ Soltero & 84 & 115 & $(109-123)$ & \\
$\quad$ Casado y Otros & 16 & 115.5 &
\end{tabular}

Hijos

$\begin{array}{rcccc}\text { Sí } & 9 & 115 & (100-119) & 0,5711 \\ \text { No } & 91 & 115 & (102-124) & \end{array}$

Áreas

$\begin{array}{ccccc}\text { Medicina } & 50 & 115.5 & (106-125) & 0,122 \\ \text { Cirugía } & 26 & 111 & (95-122) & \\ \text { Gineco-Obstetricia } & 10 & 113 & (100-118) & \\ \text { Pediatría } & 14 & 118.5 & (111-128) & \end{array}$

Año de residencia

$\begin{array}{ccccc}\text { Primero } & 44 & 116 & (100,5-126) & 0,4241 \\ \text { Segundo } & 19 & 113 & (99-118) & \\ \begin{array}{c}\text { Tercero a más } \\ \text { de egresado }\end{array} & 37 & 115 & (110-124) & \\ \text { Un año } & 44 & 116 & (103-124) & 0,4964 \\ \text { Dos años } & 23 & 114 & (103-118) & \\ \text { Tres a más } & 33 & 116 & (101-126) & \end{array}$

Taller

\begin{tabular}{rcccc} 
Sí & 10 & 118.5 & $(110-127)$ & 0,3854 \\
No & 90 & 115 & $(102-124)$ & \\
\hline
\end{tabular}

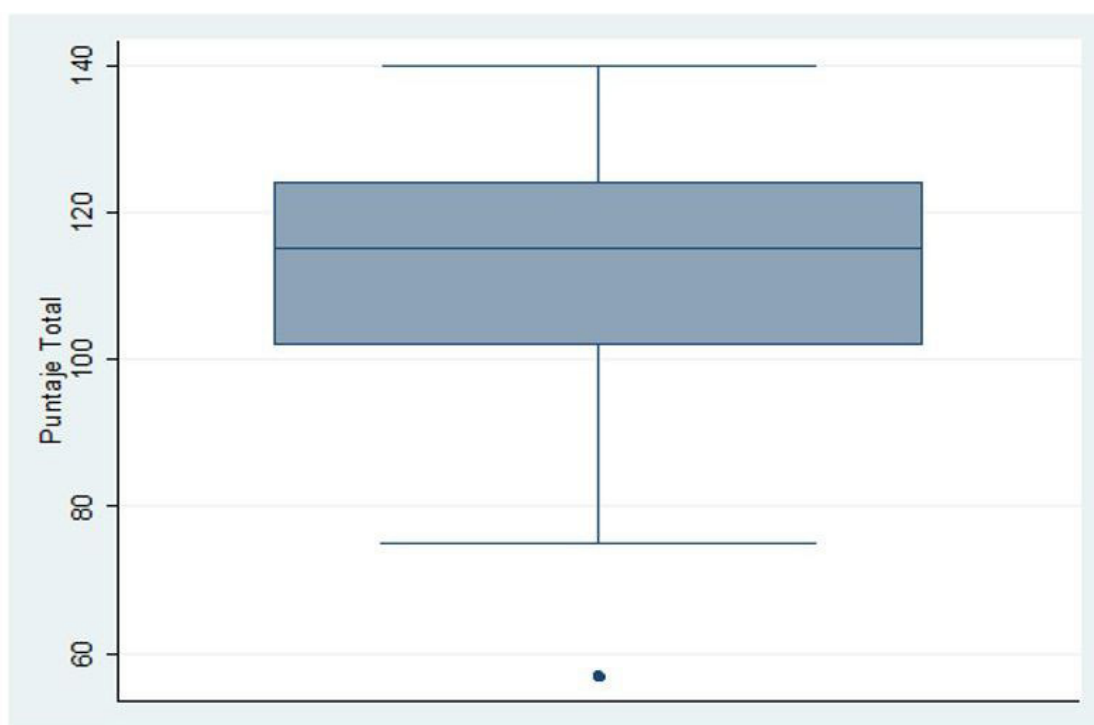

Gráfico 1. Diagrama de cajas y bigote que representa la mediana del puntaje total de empatía según la escala de Empatía Médica de Jefferson en los médicos residentes de un hospital de Lima-Perú. 


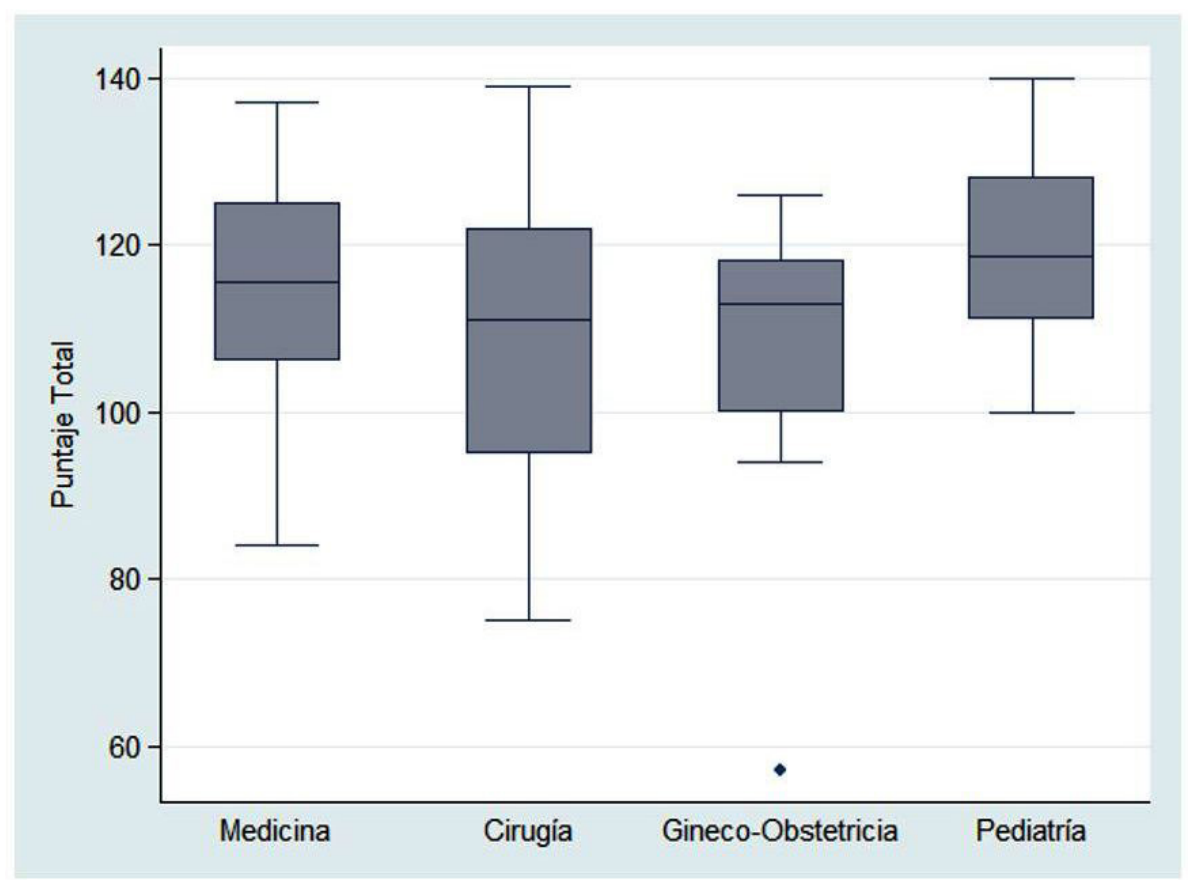

Gráfico 2. Diagrama de cajas y bigotes de puntaje total de empatía según la escala de Empatía Médica de Jefferson obtenidos según áre de especialidad médica.

Tabla 2. Estadística descriptiva y percentiles de los puntajes obtenidos según la Escala de Empatía Médica de Jefferson por los médicos residentes que realizan su especialización en un Hospital de Lima Perú.

\begin{tabular}{lc}
\hline $\mathrm{N}(\%)$ & $100(100 \%)$ \\
\hline Rango Posible & $20-140$ \\
Rango Observado & $57-140$ \\
Media & 112,88 \\
Desviación estándar & 14,51 \\
Cuartil & \\
$\quad 25$ vo & 102 \\
$\quad 50 v o$ & 115 \\
75 vo & 124 \\
Alfa de Cronbach & 0,82 \\
\hline
\end{tabular}

al área médica, 26\% quirúrgica, 10\% gineco-obstétrica y $14 \%$ pediátrica (tabla 1$)$.

El menor puntaje que se registró fue de 57 puntos, mientras que el máximo puntaje fue de 140 puntos, el mayor puntaje posible. Tanto la mediana como el puntaje máximo y mínimo de los médicos residentes de pediatría, son mayores que en los médicos residentes de las demás áreas (gráfico 2), aun así, no se halló diferencia estadísticamente significativa entre los cuatro grupos $(\mathrm{p}=0,12)$. Tampoco se encontró diferencia estadísticamente significativa al comparar los puntajes obtenidos por los encuestados según el año de residencia que estén cursando $(\mathrm{p}=0,42)$ (tabla $1)$.

La mayoría de los encuestados (90\%) no llevaron talleres o cursos enfocados en empatía durante su formación previa, siendo la mediana de quienes llevaron alguna formación en empatía 118,5 y los que no 115 , la diferencia entre ellas no fue significativa $(\mathrm{p}=0,38)($ tabla 1$)$

El test de correlación de Spearman fue utilizado para evaluar la relación entre la edad y puntaje total de los participantes, resultando en una correlación negativa 
Mayo G, et al.

Tabla 3. Nivel de empatía según la Escala de Empatía Médica de Jefferson en médicos residentes de acuerdo a las áreas del programa de especialización, años académicos y años de egresado de pregrado, de un Hospital de Lima-Perú.

\begin{tabular}{ccccc}
\hline & Bajo & Medio & Alto & p \\
\hline Área & & & & \\
& & & & \\
Medicina & $12(24 \%)$ & $25(50 \%)$ & $13(26 \%)$ & 0,401 \\
$\begin{array}{l}\text { Cirugía } \\
\text { Gineco- }\end{array}$ & $10(38,46 \%)$ & $11(42,31 \%)$ & $5(19,23 \%)$ & \\
$\begin{array}{l}\text { Obstetricia } \\
\text { Pediatría }\end{array}$ & $3(30 \%)$ & $6(60 \%)$ & $1(10 \%)$ & \\
Año de Residencia & $1(7,14 \%)$ & $8(57,14 \%)$ & $5(35,71 \%)$ & \\
$\quad \begin{array}{l}\text { Primer año } \\
\text { Segundo año }\end{array}$ & $15(34,09 \%)$ & $17(38,64 \%)$ & $12(27,27 \%)$ & 0,264 \\
$\quad$ Tercer año o & $5(26,32 \%)$ & $11(57,89 \%)$ & $3(15,79 \%)$ & \\
más & $6(16,22 \%)$ & $22(59,46 \%)$ & $9(24,32 \%)$ & \\
Años de egresado & & & & \\
Un año & $11(25,00 \%)$ & $23(52,28 \%)$ & $10(22,73 \%)$ & 0,013 \\
Dos años & $5(21,74 \%)$ & $17(73,92 \%)$ & $1(4,35 \%)$ & \\
Tres a más & $10(30,30 \%)$ & $10(30,30 \%)$ & $13(39,39 \%)$ & \\
\hline
\end{tabular}

débil (rho=-0,029), la cual fue estadísticamente no significativa $(\mathrm{p}=0,7676)$.

Cuando se clasifican los puntajes del nivel de empatía en Bajo, Medio y Alto; y se comparan con las otras variables en el estudio se obtiene que ninguna variable se asocia significativamente a alguna categoría con excepción a los años de egresado, la cual cuenta con $\mathrm{p}=0,013$ (tabla 3 )

\section{DISCUSIÓN}

La empatía médica, fue definida como un atributo cognitivo que incluye la habilidad para entender las experiencias internas del paciente, así como tener la perspectiva y capacidad para comunicar este conocimiento. Al ser aplicada en la relación médico-paciente puede mejorar la adherencia y resultados clínicos de los pacientes (18). El desarrollo de la empatía es considerado como una habilidad fundamental de la comunicación como competencia médica, la cual debe ser transversal en los programas de especialización y forma parte de los modelos de entrevista clínica estructurada vigentes (12).

La Escala de Empatía Médica de Jefferson es la más apropiada para ser aplicado en profesionales de la salud, esto por estar basado en la relación médicopaciente. Esta escala ha sido traducida a más de treinta idiomas y ha sido aplicada en más de cincuenta países alrededor del mundo, además sus distintas versiones y traducciones han mostrado tener consistencia interna, lo cual es expresado mediante el coeficiente alfa de Cronbach, que en nuestro caso fue de $0,82(18,19)$. En nuestro país, se cuenta con sólo dos antecedentes, en el año 2012 Gutierrez-Ventura et al., aplicaron esta escala en 258 estudiantes de estomatología de una universidad de Lima, obtuvieron una media de puntaje de 109.63 (DE: 13.69); posteriormente, en el 2017 Morales-Concha et al., usaron el mismo instrumento en 206 estudiantes de la facultad de medicina de una universidad del Cusco, y obtuvieron una media de $87,77(15,16)$. Esta amplia variabilidad puede deberse a que este último estudio fue aplicado estudiantes de los primeros años de la carrera, sin contacto clínico suficiente.

En nuestro estudio se encontró que la media de puntaje obtenido por los médicos residentes fue de 112,88 (DE: 14.51, mediana: 115). Nuestros puntajes resultaron ser menores a los encontrados por Hojat et al., en EUA en el primer estudio en el que se utilizó la escala médica de empatía de Jefferson en un programa de especialización, participaron 704 médicos residentes (media 120, DE 12,0, mediana 121); y los obtenidos por Di Lillo en 778 médicos residentes en Italia (media 115,1, DE 15,55, mediana 118); pero mayores que los de 229 médicos residentes 
de Corea del Sur (media 98,2, DE 12,0, mediana 99) en el estudio de Dae Hun Suh et al., Estas diferencias se deberían a influencias socioculturales como en el caso de la cultura asiática en la cual tradicionalmente la relación médico paciente es vertical o paternalista, mientras que en países occidentales el modelo horizontal está más afianzado; características de la educación médica en cada país ya que en algunos es más sólida la inclusión de la empatía en la currícula médica o el uso de modelos de entrevista clínica estructurada; también influiría el ambiente laboral de cada nosocomio $(18,20,21)$.

Respecto al nivel de empatía de acuerdo al género del médico residente, no se encontró diferencia, este hallazgo es similar al obtenido por otros autores $(22,23)$. Sin embargo, en otros estudios mostraron que las mujeres obtuvieron mayores puntajes de empatía que los varones $(3,9,24)$. Estos resultados fueron explicados por las expectativas culturales que tiene cada uno de los roles de género en la sociedad (8), esto de forma extrínseca. Y de forma intrínseca se debería al desarrollo evolutivo de la mujer, ya que se sugiere que ellas desarrollan actitudes de cuidado hacia sus hijos que concordarían con las del cuidado hacia un paciente (18).

El estado civil del médico residente no fue un factor relevante, aunque otros estudios hallaron lo contrario (9). Esto debido a que las personas casadas generalmente desarrollan determinadas habilidades como la adaptabilidad, mejor comunicación, comprensión y el manejo de emociones hacia el otro (10). Asimismo, teóricamente, la tenencia de hijos afectaría favorablemente la conducta empática y psicosocial de una persona; como menciona Park et al, en su estudio multicéntrico en Corea (9). Sin embargo, existe controversia sobre este tema. Se propone que posiblemente los médicos residentes solteros tienen más tiempo para dedicar a su bienestar personal y estar una mayor parte del tiempo con sus pacientes, desarrollando de esa manera su empatía (11). En nuestro estudio no se halló alguna diferencia significativa, probablemente se deba a una baja proporción de personas casadas o con hijos evaluadas en este estudio.

Se ha visto que la empatía médica mejora en la carrera de medicina con implementación de cursos de comunicación en la malla curricular o la aplicación de cursos de extensión $(6,9,25)$. Se puede desarrollar esta habilidad mediante programas o cursos de entrenamiento en empatía en profesionales de la salud como parte fundamental de la comunicación como competencia clínica $(26,27,28)$. Por ello, instituciones de acreditación internacionales como el CanMEDS de la Royal College of Physicians of Canada y la ACGME (Accreditation Council for Graduate Medical Education), coinciden en considerar la empatía como una habilidad en el desarrollo de la comunicación como competencia transversal en todos los programas de especialización (12).

En nuestro estudio, sólo el 10\% de los médicos residentes declararon haber llevado algún taller enfocado en empatía; además no se cuenta con datos que se puedan comparar de un antes y un después de haber recibido algún taller por el tipo de estudio utilizado. Se sebe recalcar que el desarrollo de la empatía médica como competencia de comunicación no dependerá solamente de la realización de un taller aislado sino de un programa de entrenamiento y evaluación que la abarque como parte de las competencias clínicas. Son escasos los programas de especialización a nivel nacional que implementen la formación en empatía dentro de su malla curricular.

En el Perú, se cuenta con tres modalidades de ingreso al programa de especialización médica, previa a la cual se debe realizar de manera obligatoria un año el SERUMS (Servicio Rural y Urbano Marginal de Salud). El 89\% de los encuestados ingresó por plaza libre, mientras que el $9 \%$ por plaza cautiva y $2 \%$ por destaque (29). Las distintas modalidades de ingreso se diferencian por la asociación laboral previa que tenga o no el médico residente con una institución pública. (30) Los médicos que acceden al programa de especialización mediante plazas cautivas o de destaque tienen mayor tiempo de ejercicio profesional, generalmente más de cinco años. Al analizar si la modalidad de ingreso del médico residente influye en el nivel de empatía, no se encontró diferencia significativa. Se ha visto que al aumentar la experiencia clínica de un médico con el pasar de los años disminuye la empatía, debido a la mayor carga laboral, por ende burnout y mayores niveles de depresión $(28,31)$.

$\mathrm{Al}$ analizar los años de egreso de pregrado hasta comenzar la residencia médica, se encontró que esta tenía una asociación estadísticamente significativa con el nivel de empatía. Como se mencionaba en líneas arriba, la experiencia profesional afectaría al nivel de empatía. En España encontraron que médicos residentes que no tenían más de 1 año del término de sus estudios de pregrado hasta el inicio de la residencia médica, presentaron mayores niveles de empatía que 
aquellos que toman más de un año (22). Sin embargo, cuando se observa la distribución de nuestros datos podemos apreciar que los años transcurridos desde el egreso no parecen seguir una tendencia a tener un mayor o menor nivel de empatía (tabla 3). Cabe mencionarse que cuando se compara esta variable con el puntaje total, resulta ser no significativa. Esta discrepancia en las diferencias significativas podría deberse a nuestro tamaño muestral y a la agrupación no homogénea de los participantes dentro de esta variable, por lo cual no sería relevante en la práctica.

Al igual que en otros estudios realizados en médicos residentes, no se encontraron diferencias estadísticamente significativas entre el puntaje obtenido por los encuestados de acuerdo con el año académico que se encuentran cursando $(9,22)$. Esto puede deberse a que durante la especialización aún no se han implementado programas que tengan el objetivo de desarrollar competencias en comunicación efectiva, por lo que la empatía se mantendría igual desde el inicio de la residencia médica. Asimismo, haría falta de una muestra mayor para poder generalizar estos datos o realizar un seguimiento de las personas a través de los años de formación, con lo cual se podría determinar el nivel de empatía de cada uno y evaluar si es afectada por otras variables además de esta.

Estudios previos han encontrado que médicos de ciertas especialidades suelen tener mayores niveles de empatía, entre estas "especialidades orientadas a las personas", las cuales son especialidades en las que el contacto con el paciente se da desde el principio, podemos destacar psiquiatría, pediatría y medicina interna; lo contrario sucede con médicos de "especialidades orientadas a la tecnología", especialidades que requieren de procedimientos aplicados y tienen acceso limitado a los pacientes, como radiología o cirugía $(9,18)$. Cabe destacar que estas "especialidades orientadas a las personas" son aquellas que suelen tener un enfoque integral y siguen métodos de entrevista clínica estructurada, como es el caso de los médicos especialistas en Medicina Familiar. En el Perú, los programas de Medicina Familiar generalmente utilizan el método CICAA CICAA (acrónimo de conectar, identificar, comprender problemas, acordar y ayudar), el cual además posee un instrumento que valora la relación clínica desarrollada entre el médico y el paciente(32).

En nuestro estudio se agrupó a los médicos residentes en cuatro áreas; médica, quirúrgica, gineco-obstétrica y pediátrica, observándose que, en concordancia con lo antes descrito, los médicos residentes del área de pediatría obtuvieron mayores niveles de empatía, traducido en mayor mediana, y haber obtenido el puntaje máximo y mínimo más altos respecto a las otras áreas de especialización médica, sin embargo, no se encontró diferencia estadísticamente significativa entre los grupos. Cabe mencionar que no se evaluó a médicos residentes de la especialidad de medicina familiar puesto que ellos desarrollan la mayor parte de sus actividades fuera del $\mathrm{HCH}$.

Como limitaciones de este estudio es que no permite observar la evolución de la empatía a lo largo del tiempo (22). Además, nuestra muestra sólo correspondió a médicos residentes del $\mathrm{HCH}$, principal sede docente del programa de especialización de la $\mathrm{UPCH}$, lo cual no permite generalizar los resultados a otras sedes docentes. Otra limitación es que los resultados corresponden a un auto reporte. Este evalúa sólo el primer nivel de aprendizaje según la pirámide de Miller (33). Entre las fortalezas se considera la utilización de una escala validada y usada internacionalmente, si bien no se puede generalizar, el $\mathrm{HCH}$, al ser del MINSA, puede compartir los mismos problemas que otros hospitales, por lo tanto, pudieran extrapolarse cuidadosamente estos resultados.

Recomendamos evaluar la empatía como un proceso evolutivo para lo cual serían propicios diseños de estudio longitudinales o que evalúen el nivel de empatía luego de una intervención educativa. Así mismo, recomendamos utilizar herramientas que permitan medir la empatía mediante instrumentos que evalúen niveles más altos de la pirámide Miller, sino que también consideren el punto de vista del paciente o un observador, lo que se puede lograr con métodos como el ECOE (Evaluación Clínica Objetiva Estructurada), instrumento de entrenamiento y evaluación de competencias mediante la aplicación de escenarios de simulación clínica validados, aunque este demanda más recursos $(12,25,34)$.

Como conclusiones tenemos que la media del nivel de empatía logrado por los médicos residentes del HCH de Lima Perú según la escala de empatía médica de Jefferson es de 112.88 (DE: 14.51). Los médicos del área de Pediatría obtuvieron mayor puntaje de mediana entre todas las especialidades, sin embargo, no se halló que esto fuese significativo. El nivel de empatía se asocia al número de años de egresado de pregrado que tengan. 
Utilización de la Escala de Empatía Médica de Jefferson en Residentes.

\section{Correspondencia:}

Ray Willy Ticse Aguirre.

Unidad de Educación Médica (UEM), Facultad de Medicina, Universidad Peruana Cayetano Heredia

Av. Honorio Delgado 430, San Martín de Porres, Lima, Perú.

Correo electrónico: ray.ticse@upch.pe

\section{REFERENCIAS BIBLIOGRAFICAS}

1. Rozengway H, García A, Vallecillo L. Niveles de empatía según la escala de Jefferson en estudiantes de Medicina, Enfermería y Odontología de Honduras. Rev Científica Cienc Médica. 2016;19(2):14-9.

2. Neumann M, Scheffer C, Tauschel D, Lutz G, Wirtz M, Edelhäuser F. Physician empathy: Definition, outcome-relevance and its measurement in patient care and medical education. GMS Z Med Ausbild. 2012; 29(1): 0-0. doi: 10.3205/zma000781

3. Hojat M, Gonnella JS, Nasca TJ, Mangione S, Veloksi JJ, Magee M. The Jefferson scale of physician empathy: Further psychometric data and differences by gender and specialty at item level. Acad Med. 2002;77(10):S58.

4. Larson EB, Yao X. Clinical empathy as emotional labor in the patient-physician relationship. JAMA. 2005;293(9):1100-6.

5. Shanafelt TD, West C, Zhao X, et al. Relationship between increased personal well-being and enhanced empathy among internal medicine residents. J Gen Intern Med. 2005; 20(7):559-64.

6. Esquerda M, Yuguero O, Viñas J, Pifarré J. La empatía médica, ¿nace o se hace? Evolución de la empatía en estudiantes de medicina. Aten Primaria. 2016; 48(1):1-70. DOI: 10.1016/j.aprim.2014.12.012

7. Parra G, Cámara R. Nivel de empatía médica y factores asociados en estudiantes de medicina. Investig en Educ Médica. 2017;6(24): 221-7. Doi. https://doi.org/10.1016/j.riem.2016.11.001

8. Bratek A, Bulska W, Bonk M, Seweryn M, Krysta K. Empathy among physicians, medical students and candidates. Psychiatr Danub. 2015;27 (Suppl 1): S4852.

9. Park C, Lee YJ, Hong M, et al. A multicenter study investigating empathy and burnout characteristics in medical residents with various specialties. J Korean Med Sci. 2016; 31(4):590-7.

10. Faye A, Kalra G, Swamy R, Shukla A, Subramanyam A, Kamath R. Study of emotional intelligence and empathy in medical postgraduates. Indian $\mathrm{J}$ Psychiatry. 2011;53(2):140-4.

11. Moralle M, Preston J, Chen L, Berberian W. An exploratory study of empathy in resident physicians at an Urban Medical Center. Int J Caring Sci.
2016;9(2):526-33.

12. Ticse R. El Examen Clínico Objetivo Estructurado (ECOE) en la evaluación de competencias de comunicación y profesionalismo en los programas de especialización en Medicina. Rev Medica Hered. 2017; 28(3):192-9. DOI: https://doi.org/10.20453/ rmh.v28i3.3188

13. Dossett ML, Kohatsu W, Nunley W, et al. A medical student elective promoting humanism, communication skills, complementary and alternative medicine and physician self-care: An Evaluation of the HEART Program. Explore (NY). 2013; 9(5):0-0.doi: 10.1016/j.explore.2013.06.003

14. Phillips SP, Dalgarno N. Professionalism, professionalization, expertise and compassion: a qualitative study of medical residents. BMC Med Educ. 2017;17(1):21.

15. Gutierrez-Ventura F, Quezada B, Lopez M, et al. Medición del nivel de percepción empática de los estudiantes de la Facultad de Estomatología Roberto Beltrán. Universidad Peruana Cayetano Heredia. Rev Estomatológica Hered. 2012;22(2):91-99. DOI: https://doi.org/10.20453/reh.v22i2.131

16. Morales-Concha L, Ccarita-Yucra K, MarroquinSanta Cruz JA, Atamari-Anahui N. Evaluación de la empatía en estudiantes de medicina humana en una universidad pública de la sierra sur del Perú. Educación Médica. 2018; 19(6): 327-332.

17. Garza AA, Guerrero JFG, Herrera SET, Lara FJR, Hojat M. Validación de la Escala de Empatía Médica de Jefferson en estudiantes de medicina mexicanos. Salud Ment. 2005;28(5):57-63.

18. Hojat M, Gonnella JS, Nasca TJ, Mangione S, Vergare M, Magee M. Physician empathy: definition, components, measurement, and relationship to gender and specialty. Am J Psychiatry. 2002;159(9):1563-9.

19. Hojat M, Louis D, Maxwell K, Gonnella J. The Jefferson Scale of Empathy (JSE): An Update. Popul Health Matters Former Health Policy Newsl. 2011;24(2):0-0. (Fecha de acceso 15 de marzo del 2019) Disponible en: http://jdc.jefferson.edu/hpn/ vol24/iss $2 / 5$

20. Suh DH, Hong JS, Lee DH, Gonnella JS, Hojat M. The Jefferson Scale of Physician Empathy: a preliminary psychometric study and group comparisons in Korean physicians. Med Teach. 2012;34(6):e464-468.

21. Di Lillo M, Cicchetti A, Lo Scalzo A, Taroni F, Hojat M. The Jefferson Scale of Physician Empathy: preliminary psychometrics and group comparisons in Italian physicians. Acad Med J Assoc Am Med Coll. 2009;84(9):1198-202.

22. Delgado-Bolton R, San-Martín M, Alcorta-Garza A, Vivanco L. Empatía médica en médicos que realizan el programa de formación médica especializada. Estudio comparativo intercultural en España. 
Aten Primaria. 2016; 48(9): 565-571. doi: 10.1016/j. aprim.2015.10.005

23. Hong M, Bahn GH, Lee WH, Moon SJ. Empathy in Korean psychiatric residents. Asia-Pac Psychiatry. 2011;3(2):83-90.

24. San-Martín M, Roig-Carrera H, Villalonga-Vadell RM, et al. Empatía, habilidades de colaboración interprofesional y aprendizaje médico permanente en residentes españoles y latinoamericanos que inician los programas de formación médica especializada en España. Resultados preliminares. Aten Primaria. 2017;49(1):6-12.

25. Wündrich M, Schwartz C, Feige B, Lemper D, Nissen C, Voderholzer U. Empathy training in medical students - a randomized controlled trial. Med Teach. 2017;39(10):1096-8.

26. Riess H, Kelley JM, Bailey RW, Dunn EJ, Phillips M. Empathy training for resident physicians: A randomized controlled trial of aneuroscience-informed curriculum. J Gen Intern Med. 2012;27(10):1280-6.

27. Teding van Berkhout E, van Berkhout ET, Malouff JM. The efficacy of empathy training: A metaanalysis of randomized controlled trials. J Couns Psychol. 2016;63(1):32-41.

28. Kelm Z, Womer J, Walter JK, Feudtner C. Interventions to cultivate physician empathy: a systematic review. BMC Med Educ. 2014;14:219.

29. Ministerio de Salud. Proceso de Admisión al Residentado Médico 2017: Cuadro general de vacantes por especialidad, subespecialidad, sede y universidad. Lima: Consejo Nacional de Residentado Médico; 2017. (Fecha de acceso 15 de marzo del 2019) Disponible en: http://www.conareme.org.pe/ web/Documentos/Admision2017/VAC.\%20MINSA. pdf
30. Consejo Nacional de Residentado Médico. Disposiciones complementarias. Proceso de Admisión al Residentado Médico. Lima Consejo Nacional de Residentado Médico; 2011. (Fecha de acceso 15 de marzo del 2019) Disponible en: http://www.conareme.org.pe/web/Documentos/ Admision $2011 /$ Disposiciones $\% 20$ Complementarias\%202011.pdf

31. Charles JA, Ahnfeldt-Mollerup P, Søndergaard J, Kristensen T. Empathy variation in general practice: A survey among general practitioners in Denmark. Int J Environ Res Public Health. 2018;15(3):0-0. doi: 10.3390/ijerph15030433

32. Salazar-Blanco OF, Casasbuenas-Duarte L, IdárragaArenas C, Marcela-Vélez C. Valoración de las habilidades comunicativas en la entrevista clínica de estudiantes de último año de medicina de la Universidad de Antioquia, por medio de la escala CICAA. FEM Rev Fund Educ Médica. 2014;17(4):239-48.

33. Miller GE. The assessment of clinical skills/ competence/performance. Acad Med J Assoc Am Med Coll. 1990;65(9 Suppl):S63-67.

34. Chen DCR, Pahilan ME, Orlander JD. Comparing a self-administered measure of empathy with observed behavior among medical students. J Gen Intern Med. 2010;25(3):200-222.

Recibido: 22/04/2019

Aceptado: 24/06/2019 